\title{
Clean Dirty Water - Debrief Worksheet
}

Ken Beatty Jr. ${ }^{1}$

${ }^{1}$ Journal of Conceptual and Applied STEM Education

April 28, 2020

Worksheet - Debrief

Congratulations on building your very own water filtration system! Let's talk about the results.

What happened to the dirt in the water as you poured it through your filtration system? Is that what you expected to happen? Did it improve when you swapped items? Explain.

In what ways do you think your water filter can be improved?

How many glasses of water can your filtration system tank hold (estimate)? Remember we cut a 2-liter bottle in approximately one half. How many tanks would your need to fill to produce enough filtered water for one day's worth of recommended drinking $(64 \mathrm{oz})$ ? 